\title{
"Esto de 'alternativo' no tiene nada". La construcción de la razonabilidad del uso de una medicina no-convencional desde la perspectiva de sus usuarios en Buenos Aires, Argentina
}

\section{| ${ }^{1}$ Mariana Bordes |}

Resumen: El artículo explora un aspecto del campo médico no-convencional (Ciudad de Buenos Aires): la construcción colectiva de narrativas que justifican la elección y uso de una terapia particular (reflexología) en términos de una acción razonable y válida sobre la base de su inscripción en un supuesto sentido común compartido, más allá de la adhesión al ideario alternativo. El artículo se sustenta en una investigación de tesis doctoral, de enfoque interpretativo y cualitativo, a través de entrevistas en profundidad a usuarios de esta terapia. Los resultados enfocan la centralidad de tres ejes temáticos donde esta narrativa de la razonabilidad emerge: el que se sustenta en la experiencia de los "otros significativos", bajo la retórica de la recomendación y la prueba; el relacionado con definiciones específicas de "lo real", desde las cuales la terapia se visualiza como eficaz; y otro que convalida la terapia distinguiéndola de otras opciones consideradas alteridades radicales. Se concluye que este tipo de narrativa es una de las expresiones posibles de los procesos de aculturación y asimilación que atraviesan estas terapias en contextos occidentales, posibilitando su aceptación desde amplios sectores de la sociedad más allá de la adhesión al sistema de creencias alternativo.
${ }^{1}$ Centro Argentino de Etnología Americana. Buenos Aires, Argentina. Correo electrónico: marianabordes@yahoo.com.ar 


\section{Introducción}

La pregunta en torno al por qué las personas utilizan medicinas alternativas ${ }^{1}$ (LUZ, 1997), no-convencionales o alternativas y complementarias - como las denomina la Organización Mundial de la Salud (OMS, 2002) - constituye uno de los aspectos más complejos de este campo de estudios, sobre todo por la diversidad de posturas registradas. Este escenario de variabilidad ha conducido al planteo de una dicotomía conceptual que aspira a ordenar de manera esquemática los distintos perfiles de usuarios en lo que refiere al tipo de orientación que los ligan a las medicinas alternativas (BOON et al., 1999; en BAARTS; PEDERSEN, 2009). Primero, se señala aquellos casos en que la biomedicina "falla", afirmando que la incursión en prácticas no-convencionales se desprende de una actitud pragmatista que intenta solamente obtener una respuesta instrumental (la cura, el alivio, etc.) ante la irrupción de una dolencia. En contraste, el segundo perfil describe la adhesión a las medicinas alternativas signado por una atracción hacia los sistemas de creencias que fundan estas terapias, lo que remitiría a una adhesión fuerte al ideario - o "ideología” - en cuestión (LEISER, 2003).

Este artículo no busca zanjar este dualismo, si bien puede constituir un aporte para su revisión. Este trabajo ahonda en una línea argumentativa delineada por los usuarios de una terapia alternativa (la reflexología podal ${ }^{2}$ ), en cuyo contexto se define la elección de la terapia como una acción no sólo congruente con la propia situación biográfica (necesidades de salud, redes sociales) y con las concepciones acerca del cuerpo y lo que un recurso terapéutico "debe ser" para ser valorado. Sino como una acción razonable, más o menos válida para un $x$ cualquiera; es decir, como parte de una comprensión de sentido común que se supone mutuamente compartida (SCHUTZ, 2003; GIDDENS, 2007).

El interés de estos enunciados radica en que, desde la propia lógica de su formulación, expresan un rechazo hacia la necesidad de adscribir a una ideología alternativa para denotar el carácter aceptable de la práctica. Además, impiden pensar en términos exclusivamente utilitaristas, lo que ya ha sido cuestionado desde la literatura de las medicinas alternativas, por suponer una elección y uso terapéutico desde un vacuum cultural. Esta perspectiva pone de manifiesto que, más que pensar en términos de un "adentro" y un "afuera" del campo alternativo, deben indagarse las formas en que estas terapias forman parte del horizonte sociocultural de las personas, aún si no adhieren a teorías y/o preceptos formales. 
En este sentido, retomamos el planteo de Judith Fadlon (2005), quien sostiene que estas medicinas - o al menos algunas variantes de las mismas - han logrado atravesar un proceso de asimilación/aculturación que las ha tornado menos exóticas y más familiares para amplios sectores de la población.

El objetivo del artículo es analizar la construcción narrativa de la razonabilidad en torno al uso de la reflexología que esgrimen sus usuarios, pero de una razonabilidad con aspiraciones de ser aceptada por "los otros" en general. Nos referimos al trabajo activo que emprenden los actores sociales a la hora de generar una "explicación acorde con lo que es generalmente reconocido, en su ambiente social, como razonable" (GIDDENS, 2007, p. 141). Nuestro planteo es que este tipo de razonabilidad se articula con un conocimiento práctico de la gestión del malestar (VISACOVSKY, 2009) que si bien pondera a la reflexología como terapia no-convencional, no termina de definirla como alteridad radical. Para ello, desarrollamos tres ejes (cada uno correspondiente a un apartado), que emergen en los relatos de usuarios a la hora de analizar las justificaciones de la elección y uso de la reflexología en tanto acción válida para el alivio del malestar y/o cuidado. En el primer apartado, subrayamos la relevancia otorgada a la experiencia de sus "otros significativos", bajo la retórica de la recomendación y la prueba. Luego, nos adentramos en distintos aspectos legitimantes de aspiración empirista (desde el carácter "tangible" del masaje, las homologaciones que se operan entre la reflexología y la biomedicina). Por último, ahondamos cómo se construye la definición de la terapia por fuera de la alteridad terapéutica posición ocupada por otras opciones - sobre la base de una serie de valoraciones relacionales entre lo estigmatizado y lo legítimo.

Este artículo se desprende de un trabajo de investigación doctoral de enfoque interpretativo y cualitativo (MINAYO, 2009) financiado por el Consejo Nacional de Investigaciones Científicas y Técnicas (CONICET), focalizado en las dinámicas de construcción de sentido terapéutico en el espacio social de la reflexología. La misma cuenta con un corpus de 48 entrevistas en profundidad a usuarios y especialistas, además de observaciones participantes realizadas durante el período 2007-2011 principalmente en dos institutos de reflexología sitos en Ciudad de Buenos Aires, Argentina. La noción de razonabilidad apareció como categoría emergente durante el análisis de los datos, mencionada en el marco de dos ejes de indagación: (a) la reflexión respecto de la elección terapéutica (¿por 
qué la elegiste?, ¿por qué esa terapia y no otra?) y (b) las narrativas que hacen a la interpretación de su eficacia. En este artículo, nos centramos en la perspectiva de los actores sociales poniendo el acento en lo que "dicen" y no en lo que "hacen". Lo que ha respondido a ciertas características del objeto de estudio, como el carácter privado de las sesiones terapéuticas. Por eso, si bien la investigación se había planteado desde un enfoque etnográfico, finalmente optamos por el estudio de caso etnográfico (STAKE, 1994) y la narrativa de casos (CRIVOS, 2007) para organizar trabajo de campo y análisis.

\section{El punto de partida: la "prueba"}

En nuestro trabajo, lo primero mencionado por los usuarios al explicitar su asistencia a una consulta reflexológica está signado por la expectativa de atender algún padecimiento o, al menos, se señala algún tipo de inquietud respecto de molestias que se quiere gestionar para evitar problemas potenciales. Aquí, coincidimos con Baarts y Pedersen (2009), en tanto el malestar suele emerger como tópico central cuando se trata de explicar el uso de la reflexología como terapia - a diferencia de otras alternativas como el mindfulness, buscadas para la mejora general de la salud o el auto-desarrollo (2009, p. 722). Los motivos pudieron clasificarse en: (a) dolores y molestias de índole óseo-muscular crónicas y agudas - que van desde el señalamiento de categorías nosológicas de la biomedicina - artritis reumatoidea, escoliosis, lumbalgia; hasta padecimientos más difusos definidos como "contracturas", "dolores", "puntadas". Aquí algunos informantes refirieron problemas posturales asociados al ámbito laboral; otros situaciones psico-emocionales como el estrés, la ansiedad y los "nervios", percibidos como ligados con - sino como causa de - la formación de las tensiones y contracturas; (b) cefaleas, pérdida de equilibrio y mareos; (c) dolencias gastrointestinales; (d) otras personas decidieron asistir debido a dolores y/o afecciones en pies y/o piernas, lo que se corresponde con la parte del cuerpo en la que hace foco esta reflexología (pies); (e) otros la utilizaron como terapia válida para tratar malestares psico-emocionales. Aquí, la reflexología se mencionó como instancia de acompañamiento en circunstancias percibidas como difíciles, por ejemplo, una cirugía o accidente. O bien, como opción que complementa e incluso reemplaza al psicoanálisis. Sin embargo, excepto en un caso señalado como motivo específico, los entrevistados mencionaron el malestar psico-emocional 
como concomitante a un tratamiento enfocado en lo físico, lo que muestra la dificultad de separar de modo tajante la dimensión física y la emocional; (f) como práctica para mejorar y/o preservar el estado de salud general.

Si bien estos padecimientos aparecen como instancias objetivas susceptibles de ser definidas como los motivos porque $e^{3}$ que fundan el sentido de la acción vista retrospectivamente (SCHUTZ, 2003), es preciso realizar una operación de contextualización si se intenta comprender en términos interpretativos los componentes cognitivos, valorativos y morales puestos en juego al justificar las elecciones médicas.

En primer lugar, un elemento legitimador de la elección - tanto en usuarios que nunca habían tenido una experiencia previa en este campo terapéutico, como en aquellos que sí - es la referencia a una recomendación desde el entorno cercano, considerando el contexto laboral, familiar, terapeutas tanto del circuito alternativo como biomédicos - médicos alópatas, psicólogos. Esto no excluye que las personas expliciten su acercamiento como resultado de una trayectoria de búsqueda - que incluye el rastreo a través de internet, medios gráficos -, como parte de la participación activa en el circuito de prácticas terapéuticas alternativas (MAGNANI, 1999) o de un encuentro considerado fortuito. No obstante, la recomendación por parte de los "otros significativos" del individuo en cuestión (CANT; SHARMA, 1999) no sólo sitúa a la elección de la terapia - muchas veces desconocida por el usuario como opción viable dentro del horizonte de posibilidad social y cultural, sino que brinda un soporte o marco de validez en tanto ya fue probada: (a) por una persona "de confianza", (b) con más o menos éxito y (c) sin provocar perjuicio constatable. La recomendación de practicantes terapéuticos entre conocidos constituye un patrón frecuente cuando se analiza las trayectorias de las personas en búsqueda de la salud (SHARMA, 1992), lo que ha sido analizado destacando la relevancia de las redes sociales de cuidado (FREIDIN; TIMMERMANS, 2008).

Pues bien, desde la perspectiva interpretativa aquí adoptada, no analizamos este tópico en sí mismo, sino como parte de la construcción de un sentido práctico de la gestión del malestar (VISACOVSKY, 2009) que se supone más o menos compartido - o pasible de serlo - al denotar esquemas interpretativos considerados comunes respecto de las circunstancias en las cuales se torna aceptable apelar a un recurso terapéutico no-convencional. 
La recomendación como instancia legitimadora opera al abrir un umbral de acción definido por una retórica de la prueba, que ocupa un lugar central en los relatos como modalidad de explicación de la elección terapéutica. Esto implica que la recomendación no funda la creencia en la potencial infalibilidad de la propuesta; sino que ofrece un punto de partida habilitante. "¿Por qué no?”, "vamos a ver de qué trata", o el más radical "no tengo nada que perder" denotan una retórica desde la cual la ausencia de resultados y consecuente desestimación de la práctica son contemplados como posibilidades a la hora de elegir una terapia. Esta instancia de "prueba" puede interpretarse retrospectivamente como signada por el entusiasmo, la curiosidad y la atracción, y/o por algún grado de escepticismo ante lo considerado como desconocido o alejado culturalmente. En particular, este "voy a ver qué ocurre" aparece con frecuencia cuando se trata de una dolencia recurrente y/o puntual que no pudo ser conjurada por los recursos médicos disponibles - en los cuales los sujetos se encuentran enculturados; o cuando no se los considera un recurso suficiente.

El cansancio ante los fracasos previos, así como la persistencia de un dolor, contribuyen a moldear narrativamente la autoevidencia respecto del sentido que revistió - y reviste - aceptar las recomendaciones del entorno y tentar con una opción "diferente". Este es el caso de dos usuarias cuyos relatos citamos. Una, con contracturas de espalda a raíz de las condiciones posturales que le imponen sus actividades cotidianas - trabajo de gabinete con computadora - quien asiste a la especialidad biomédica que considera más cercana (traumatología). No obstante, si bien la credibilidad en la biomedicina se reactualiza a nivel de un diagnóstico considerado certero, la propuesta terapéutica ofrecida - kinesiología - se interpreta como no exitosa. Lo cual es referido, en este relato y en muchos otros: la falencia de la fisioterapia biomédica en la gestión de los padecimientos del estilo de vida (BORDES, 2007). La otra usuaria, una persona mayor que sufre de mareos que desde la neurología y la otorrinolaringología no puede resolver.

Fui al traumatólogo, me sacaron la radiografía, tenía la columna rectificada. Kinesiología, como lo convencional [...] hice un par de sesiones [...]. Hice las primeras diez que te dan, pedí como otras diez extras y no tuve resultado, seguí igual. Y una amiga de mi mamá estaba yendo a reflexología, la conocía hacía un tiempo y me dijo "bueno andá a probar”, qué sé yo, mi mamá había ido primero, también porque le dolía esto, lo otro y fue a probar. Y yo fui, y bueno, el primer día fui medio entre escéptica y diciendo bueno, "mal no me va a hacer" (F., usuaria de reflexología y kinesiología). 
Mi cuñada me dice ‘¿por qué no venís a lo de Enrique?’ yo sabía que ella iba, que le

había hecho mucho bien respecto de problemas que ella tenía de huesos, entonces me llevó... [...] entonces comencé, fui como una experiencia más, dije 'voy a ver qué pasa', aunque con las mejores referencias respecto a mi cuñada. Pero bueno, para mi es algo, me vieron tantos médicos... me hicieron tanto" (N., usuaria de reflexología)

El "probar" con la reflexología no constituye, claro está, una consecuencia necesaria del fracaso de la medicina ortodoxa en el tratamiento de determinada dolencia (MacGREGOR; PEAY, 1996), a pesar de recalcarse el agotamiento de todas las posibilidades brindadas desde el campo "convencional" como argumento validante de las búsquedas en el campo alternativo. En el trabajo de campo, se constata un continuum de argumentaciones posibles, entre las cuales se observa la concepción de que toda dolencia requiere complementar la terapéutica biomédica con otra que aborde los aspectos psicoemocionales de la persona; o que determinados malestares - por sus características intrínsecas - requieren de tratamientos diferenciales, no centrados exclusivamente en un modelo bioquímico, siendo la reflexología un recurso viable para gestionar molestias que no constituyen un problema desde el marco de inteligibilidad de la biomedicina.

Sin embargo, estos argumentos son potencialmente interpretables como parte de una "creencia" propia del ideario alternativo. Mientras que el fracaso de la terapéutica biomédica se constituye en un elemento narrativo fuerte a la hora de oficiar como justificador de la acción en el marco no-convencional en tanto puede resultar válido de manera más o menos generalizada, al menos para aquellos que hayan atravesado por una situación similar. Desde ambas posturas, empero, la recomendación opera por igual como dispositivo social que permite tornar más "familiar" una terapia desconocida.

\section{La legitimidad de lo "real"}

A la hora de dar cuenta de los motivos de utilización de la terapia, las narrativas hacen referencia al /a los resultado/s obtenido/s, lo que reviste especial centralidad en lo que concierne a la construcción de una justificación de la validez frente a los otros. La manera en que los actores sociales interpretan la eficacia de un determinado recurso médico, así como el modo en que la fundamentan, depende de múltiples aspectos que - como señala Barry (2006) - exceden la retórica de la evidencia, basada en la estandarización de las pruebas, que se esgrime en el 
campo médico ortodoxo ${ }^{4}$. En este sentido, la lectura lega del "éxito" terapéutico en el marco alternativo ha sido estudiada teniendo en cuenta el entrelazamiento de la dimensión técnica y la ritual que se opera a nivel de la situación terapéutica (MALUF, 2005; GALE, 2011); las experiencias vividas desde una lectura fenomenológica (ADAMS, 2002), el registro de la presencia del cuerpo en términos perceptivos (bodily awareness) (BAARTS; PEDERSEN, 2009); las consecuentes resignificaciones corporales que redundan en cambios a nivel de las definiciones identitarias y del self (McGUIRE, 1988; SOINTU, 2006). Sin dudas, estos planteos detallan de manera pormenorizada los rasgos principales de la epistemología alternativa y su modo de "funcionamiento" - centrado en una mirada sobre la persona en tanto totalidad, que define criterios de diagnóstico y tratamiento individualizados (JOHANESSEN, 1999), que son recuperados por los usuarios, sobre todo, aquellos "comprometidos" con las respectivas terapias (BARRY, 2006, p. 2.654). Reconociendo estos planteos, aquí nos interesa solamente explicitar el hecho de que, más allá del grado de adhesión al ideario de creencias alternativo que detenten, las explicaciones legas mantienen un nivel general donde el esfuerzo comunicativo radica en demostrar el carácter "real" y "objetivo", en el sentido de una eficacia comprobable para cualquiera, aún cuando la constatación remita a un nivel particular.

En primer término, cabe destacar la relevancia de la experiencia en los relatos analizados, la cual se enmarca en una lógica del testimonio personal de la eficacia de la técnica. Aquí, recuperamos la definición de experiencia corporal que esgrimen Baarts y Pedersen (2009) citando a Gendlin (1997), que concierne la referencia directa verbal que realiza el individuo respecto de lo que fenomenológicamente se le presenta, es decir, lo que percibe. Todo lo cual implica una referencia selectiva en torno a lo que es considerado significativo en un contexto terapéutico, en tanto los actores sociales operan un ejercicio reflexivo de índole retrospectiva que intenta reconstruir las vivencias del pasado - más o menos cercano - para brindar una interpretación de la acción en el presente. Al igual que lo constatado ya en la literatura de ciencias sociales, los relatos analizados expresan que la evidencia de que la terapia "funciona" no se desprende necesariamente del alivio del síntoma por el cual se concurre a sesión. Generalmente, se menciona el hecho de haber constatado que "algo sucede" durante la aplicación técnica manual, 
atribuyéndose una relación de causalidad entre el estímulo en los pies y algún tipo de registro, sobre todo, a nivel físico y/o emocional - aunque también se mencionan cambios en el marco de las relaciones interpersonales del individuo, lo que presupone un lapso más prolongado de tratamiento, como bien consigna Barry (2006, p. 2.651). La relación experiencial estímulo-efecto adquiere más peso que cualquier intento de explicación formal:

Lo que yo sentí era que [...] me toca un lugar que tenía que ver con la cabeza y me re dolió, le pregunté ‘qqué es eso?', y me dijo 'este es el punto de la armonía'. Me gustó que lo que me decía tenía un asidero en la realidad, después, me sentí bien, dormí bien a la noche, me relajó. [...] (P., usuaria de reflexología, reiki, yoga).

Y lo sentís. Sentís como se te va abriendo los esfínteres, no sé nada de anatomía, el intestino grueso no sé lo que se te abre. Pero sentís cosas re locas, re locas, que realmente no sabías que estaban en tu cuerpo y que realmente las podías sentir (Y., usuaria de reflexología y homeopatía).

El espectro de estas constataciones es amplio, aunque brevemente podríamos referir: bostezos, frío/calor, transpiración, eructos, ahogos; lo que se interpreta generalmente en términos de expulsión de aquello que "sobra" en el cuerpo, avalando una lógica terapéutica sustractiva del malestar (LAPLANTINE, 1999, p. 88). La visión de imágenes/colores, llanto, risa, enojo, tristeza, la constatación de que la condición por la que se acude a la terapia empeora, o el retorno de una molestia o dolor pasados, aparecen muchas veces interpretados como signos de que el cuerpo tiene una "memoria" que convierte las experiencias vividas en marcas fijadas en el cuerpo (SAIZAR, 2009) que "salen a la luz" a partir del estímulo manual del masaje. La relajación, sensación de paz y tranquilidad, son leídos como efectos de la armonización corporal que se gesta como resultado de esta expulsión; o como efectos directos del masaje, generando movilización, distensión de los músculos y consecuente bienestar.

Las características propias de la reflexología conllevan una serie de relaciones significativas suplementarias. En primer término, la lectura que asigna un valor de realidad objetiva al efecto que se registra a partir del toque manual como legitimador de la terapia, se asocia a la dimensión "tangible" que pone en juego la reflexología. En Bordes (2012), señalamos que este tipo de aseveración adquiere sentido en términos relacionales, en la medida en que opera una distinción respecto de todas aquellas terapias consideradas "sutiles", esto es, que no suponen 
el contacto manual con el cuerpo del paciente al manipular energía de manera exclusiva. El par conceptual tangible-sutil se concretiza muchas veces en la oposición entre la reflexología y el reiki:

Cuando ella me hacía reiki a mí me costaba creer. Debe ser porque con reflexología vos sentís el masaje, en cambio en el reiki es... yo sentía que los pelos ¿iviste cuando te peinás y te queda así? [electrizado] [...] yo creo más en lo, como en lo tangible, en eso me cuesta creer [...] a mí me cuesta creer que puedo recibir de algo que no me está tocando (D., usuaria de reflexología y kinesiología)

Como es posible apreciar, la ausencia de contacto manual lleva a descartar la selección del reiki como recurso terapéutico, a la vez que conduce a interpretarlo como una práctica que exige contar con un marco interpretativo que avale este tipo de acción sutil sobre el cuerpo. De hecho, en algunos fragmentos de la entrevista aparece explicitada la reflexión de que el rechazo al reiki se debe al propio desconocimiento acerca de terapias consideradas culturalmente distantes, antes que a un atributo de la terapia en sí misma ("el que hace reiki a mi no me llama ni me llega, por ignorancia también puede ser, como cuando los chicos que les decís 'iquerés comer esto?' 'No, no me gusta' ‘lo probaste?' 'No', '[entonces] ¿cómo sabés que no te gusta?'”). En contrapartida, el valor de lo tangible se corresponde con la dimensión física de la corporalidad que se expresa a través del masaje, lo que además es codificado en términos de mayor cercanía cultural, comparándose con las prácticas corporales "ya conocidas", como la fisioterapia biomédica -reiteradamente referida al señalar el ámbito de competencia que la medicina convencional comparte con la reflexología en términos de intervención sobre el cuerpo (BORDES, 2007)-. Esta cercanía se constata no sólo a nivel de la implementación técnica manual, sino asimismo a nivel del tipo de efectos que produce. Así, más allá de que puedan mencionarse sensaciones o emociones que generan sorpresa por su emergencia repentina y/o inesperada -como la irrupción del llanto durante el masaje-, las narrativas que sostienen la razonabilidad de la terapia en el sentido aquí utilizado, enfatizan el hecho de que el beneficio de la terapia no representa ningún efecto por fuera del terreno socioculturalmente cercano.

- Hay un prejuicio muy grande, se lo asocia con la magia, con la astrología, como fuera que vas a una tarotista.

Investigadora: ¿Y a vos qué te parece que es? 
- A mi me parece que en un punto está tomado como alternativo porque no es lo comúnmente aceptado. Si es algo totalmente tangible y de alternativa no tiene nada, qué sé yo, nunca se me dieron vuelta los ojos [...] Esto es totalmente tangible, te digo por haber vivido resultados y haber recomendado por ahí a mi mamá o a una amiga que haya ido y le haya solucionado algo... Los ruidos en la panza yo no los invento (E., usuaria de reflexología, kinesiología, psicología)

Este fragmento denota una construcción del aspecto tangible de la terapia en virtud de su carácter empíricamente comprobable -es decir, la existencia de efectos anclados en la realidad del cuerpo físico-: en su caso, la reducción del dolor y la constatación de que, al tocarse un punto en los pies, se registra un correlato en el sistema digestivo ("ruidos en la panza"). Para ello, esboza dos líneas argumentativas en oposición a lo que visualiza como las críticas que circulan hacia los abordajes terapéuticos no-convencionales en general. Primero, excluye de la reflexología todo efecto extraordinario, místico, esotérico o paranormal (LEISER, 2003) que traería aparejada una terapia "auténticamente alternativa" -término que aquí es utilizado en oposición a convencional- que la usuaria explicita al decir "nunca se me dieron vuelta los ojos". Esto es, efectos clasificables en términos de lo que la antropología definió como estado alterado de consciencia o de experiencia numinosa (OTTO, 2005). Segundo, discute con la posibilidad de que se le atribuya, por ejemplo, un sentido de efecto placebo al indicar que, tanto ella como otras personas de su entorno, constataron efectos "reales". El relato citado explicita un aspecto habitual en las narrativas de la razonabilidad de la elección y uso de la terapia en los términos conceptualizados: el de oponer la propia experiencia vivida a los discursos detractores de las "alternativas", esgrimiendo argumentos considerados potencialmente válidos para éstos últimos. Aquí se pone en juego una discusión acerca de lo que es real y verdadero para el ámbito de la realidad médica, sobre todo al defender la eficacia de las técnicas al interior de un enfoque terapéutico - la reflexología- que se encuentra por fuera del campo ortodoxo de la atención de la salud. Este aspecto no-reconocido de la terapia a nivel oficial es algo sabido por los entrevistados. Las posibles críticas y descalificaciones que esto puede acarrear por parte de personas no familiarizadas con este campo terapéutico, refuerza entonces la explicitación de tomas de posición que intentan demostrar las destrezas - propias y del entorno- a la hora de actuar.

A las interpretaciones que argumentan la legitimidad de la terapia reflexológica en términos del carácter tangible de su intervención, se suman otras que 
esgrimen elementos de autoridad relativos a sistemas expertos e instancias de reconocimiento oficial. Aquí surgen referencias como "es un estudio, una carrera" -reforzando la idea de que requiere una formación formal para ser ejercida-; "mi prima que es médica la 'banca' [la defiende]" -haciendo alusión a personas que, al interior del campo científico, consideran la validez de la terapia, de algún modo autorizando su uso-; "tiene también como toda una teoría lógica, super lógica me parece a mí, por lo que yo estudié, que la sustenta [...] desde lo cientifico digamos [...] tiene una parte real de interconexión de nervios a través del cuerpo". Esta última frase, planteada por un usuario que se formó durante algunos meses como practicante de reflexología, consigna un doble movimiento: (a) el de la suposición lega de que la repercusión en otra parte del cuerpo de un estímulo aplicado en el pie, se funda en algún soporte muscular o nervioso - y (b) la recuperación del discurso de un sector de los profesionales reflexólogos -en Argentina, representado en la Asociación Argentina de Reflexólogosde una teoría biomédica (neuromatrix theory) que afirma que la estimulación podal repercute a nivel del sistema nervioso central y del endocrino, generando un efecto a nivel cerebral - por ejemplo, disminución del dolor (MELZACK, 1999 en STEPHENSON; DALTON, 2003, p. 182). Cabe señalar que estas referencias a dispositivos legitimadores "oficiales" son esgrimidas tanto por los usuarios menos comprometidos con el ideario alternativo, como por aquellos que sí lo están; más allá de que en ciertos apartados del relato se otorgue más o menos peso a saberes no-ortodoxos (como la medicina tradicional china) o a nociones extranjeras relacionadas, como meridianos, chi, prana, profundamente refiguradas bajo la denominación occidental de "energía".

\section{Trazando los límites de la razonabilidad terapéutica}

En este apartado nos centramos en un tercer eje que encontramos a la hora de analizar el esfuerzo de las narrativas de la razonabilidad por sustentar la validez y coherencia de la elección y uso terapéutico no-convencional desde un supuesto sentido común compartido: el que remite a la razonabilidad del uso de la reflexología por oposición a otras terapias, considerando un aspecto principalmente moral: el que implica la clasificación de las personas - y de sísegún la posición adoptada respecto de terapias que son diferentes, pero no tan distintas al planteo convencional. Aquí, es relevante recorrer dos ejes valorativos: 
(a) el que supone una lectura negativa respecto de aquellos individuos que rechazan las "alternativas" y (b) el que define como una destreza la posibilidad de abrirse a nuevas opciones médicas. Nos interesa trazar una postura expresada de modo recurrente por muchos entrevistados: la idea de que la preferencia por esta opción de cuidado no es equivalente a definirse como consumidor de terapias alternativas, aunque sí supone ciertos rasgos de la persona en cuestión.

Estos ejes valorativos se sustentan en dos elementos encontrados en el campo. En primer lugar, una lectura de que el rechazo radical hacia una terapia como la reflexología es el resultado de una actitud negativa respecto de opciones terapéuticas diferentes pero potencialmente útiles. Lo que generalmente - se interpreta - no supone fundamentos válidos sino una serie de prejuicios basados en el desconocimiento y/o la apropiación de discursos sociales descalificadores. Las referencias que encontramos en los relatos remiten principalmente a la lectura de estas terapias en términos de engaño - "mentira", "blef", "chantada" - o la idea de que funcionan bajo un efecto placebo; así como las asociaciones con la magia - y referentes asociados como la astrología, el tarot, la adivinación-, y, en menor medida, a la brujería. Cabe destacar que, respecto de las dos primeras referencias, el foco está puesto en las posturas generalizantes que descartan $a$ priori toda posibilidad de verificación; mientras que las dos últimas se consideran una operación errónea de clasificación, distorsionando aquello que tienen de específico las "alternativas" al asimilarlas a prácticas que no tienen ningún aspecto en común. De este modo, las narrativas expresan un reconocimiento de los postulados del rechazo de las medicinas no-convencionales, clasificando a los individuos que los vehiculizan en un continuum que va desde la incredulidad radical (es decir, cuando no les atribuyen ningún efecto posible) hasta la posibilidad de creer en "algo" que funciona como soporte de la terapia pero que, como no se es capaz de explicarlo o de comprenderlo, culmina en un rechazo. He aquí un fragmento donde una usuaria habla de la experiencia de un familiar, quien acude a un homeópata y, a pesar de obtener resultados óptimos, decide abandonar la terapia esgrimiendo como argumento un vínculo con la brujería.

E: [mi abuela] muy hipocondríaca [...] mi mamá había intentado llevarla, ella iba al homeópata y cuando le empezó a dar resultado empezó a quejarse y después la volvieron a llevar al médico alópata

Investigadora: -¿Por qué? 
E:-Claro... porque también estaba grande y se pone complicada la cosa. Como que estaba mejor entonces tenía menos atención, menos miradas encima... entonces empezó a decir que [el homeópata] no le caía bien. Una vuelta dijo que había soñado que era un brujo [...] Yo no sé si adrede o desde una mentalidad muy cerrada, por ahí un poco más de alguien que tampoco estaba en un momento cronológico para abrirse a cosas nuevas (E., usuaria de reflexología y kinesiología)

La crítica de los críticos de las "alternativas" conlleva muchas veces una mirada centrada en lo que se vislumbra como una incapacidad: el rechazo ante nuevas experiencias. Esta cerrazón - considerada como exenta de fundamentos válidos y como una actitud a priori que impide la elección razonable de opciones de utilidad potencial - se contrapone a una representación acerca de la propia capacidad de agencia en términos de una "mente abierta", lo que es valorado de modo positivo. Una usuaria - médica alópata - comenta una anécdota durante una estadía en el campo, a partir de la cual destaca su "apertura" hacia las prácticas no-convencionales:

La semana pasada estaba con mis primas y a mi sobrino le había salido un sarpullido. Entonces le digo: 'yo le pondría una cremita, una cortisona [...]'. Y una amiga de ella dice 'acá en el campo lo que hacen es batir clara de huevo y se lo ponen cuando hace espumita'. Mirá, yo soy una mujer de ciencia, yo necesito ver para creerte [...] entonces mi prima se lo hizo y al día siguiente el gordo estaba fantástico [...] o sea, yo soy una mujer de ciencia pero estoy abierta a ver los mitos de campo, ¿cuántos mitos de campo hay respecto de la salud? (C., usuaria de reflexología y masajes).

La posibilidad de aprender, de acceder a otras verdades, de "ver" lo que antes no se podía ver, son ideas planteadas en este contexto. Como señala un usuario:

Algunas personas que hacen cosas alternativas me demostraron que hay otras verdades... o que lo que se nos vende con la medicina alopática no es toda la verdad [...] cuando descubrí la acupuntura dije 'puede ser que haya otras cosas que sirvan'”.

Aquí, lo diferente no es leído como peligroso, sino que emerge como una dimensión de lo posible que vale la pena explorar si se constata su eficacia en algún sentido. Este aspecto se constituye en el segundo punto que se articula con la lectura negativa de la "cerrazón" respecto de lo nuevo: una valoración positiva en torno de la incursión en prácticas no-convencionales, potencialmente beneficiosas. Aquí, dialogando con la sociología informada hermenéuticamente (GIDDENS, 2007) podemos consignar que se trata de una elaboración reflexiva que opera atribuyéndose para sí (y para aquellos que obran igual) una competencia particular a la hora de actuar: la disposición de trascender los 
límites de "lo conocido". Claro está, esta capacidad suele acompañarse por un consenso acerca de que estas terapias "funcionan", involucrando - al menos alguna experiencia propia y/o ajena que lo valide. No obstante, la capacidad de decisión es vislumbrada como un atributo personal y, por ende, producto de la propia destreza a la hora de evaluar lo que es útil para sí.

Para cerrar este apartado, podemos destacar que - incluso esta postura de "mente abierta" - supone una delimitación en torno a las propuestas terapéuticas que no fueron y/o no serían elegidas bajo ninguna circunstancia. Para los usuarios entrevistados, podemos señalar dos extremos en un continuum de posturas donde se aceptan y excluyen distintas opciones. Por un lado, una visión que sitúa por fuera del horizonte de posibles a ciertas terapias religiosas, tradicionales e incluso algunas alternativas ${ }^{6}$ - "curandero", "evangelistas", "pai umbanda", "flores de Bach". Estas no son consideradas fiables o "serias" en la medida en que se las contrapone a la dimensión objetiva atribuida a la reflexología, de acuerdo a su carácter tangible y/o potencialmente validable en términos biomédicos, lo que se opera: (a) adjudicándoles una dimensión peligrosa o (b) un rechazo desde el argumento del desconocimiento, enfatizando la distancia cultural respecto de la práctica. Por otro lado, se encuentra el caso del rechazo hacia posturas radicalizadas dentro de las propias medicinas alternativas, tratándose en algunos casos de usuarios que cuentan con una extendida trayectoria en el denominado circuito alternativo (MAGNANI, 1999). Aquí, la crítica radica en las pretensiones religiosas - sobre todo de conversión - de una práctica que desde la perspectiva de los actores sociales, es buscada por su eficacia terapéutica, sea cual fuere la interpretación de esta eficacia.

[La clase de yoga] estaba acompañada de una lectura de algo cuasi religioso, supuestamente filosófico de bajada de línea de cómo vivir [...] No me sentía a gusto con eso. (E., usuario de yoga y reflexología).

[Con mi homeópata] vuelvo, me peleo, vuelvo, se fue volviendo más budista, más extremo y te tiraba cosas delirantes y me ponía muy nerviosa [...]. No es muy fácil ser un yogi en Buenos Aires. (Y., usuaria de homeopatía y reflexología).

Este distanciamiento respecto de un compromiso "extremo" con ciertas creencias y prácticas alternativas ha sido ya señalado. Por ejemplo Carozzi (2000, p. 106-107) consigna la tendencia a evitar las intervenciones de otros en el propio accionar, suprimiendo las identificaciones con modelos de creencia 
específicos. En el caso de nuestro trabajo de campo, la referencia estuvo puesta en la preferencia por un tipo de terapia más "relajado", en el sentido de más ajustado al estilo de vida occidental y urbano, cuya organización del tiempo se encuentra signado por la cultura del trabajo capitalista - en muchas ocasiones de carácter flexible e inestable. En este sentido, se pondera la capacidad de adaptabilidad de $\mathrm{la} / \mathrm{s}$ terapia/s a distintos perfiles de usuarios con necesidades diversas.

\section{Conclusiones}

El objetivo del trabajo fue mostrar algunos elementos narrativos que enfatizan los esfuerzos comunicativos llevados a cabo por usuarios de una terapia alternativa - la reflexología - para justificar la elección y uso de la misma en términos de una acción sensata y razonable. En los relatos recabados frecuentemente se apeló a nociones comúnmente asociadas al ideario alternativo -energía y armonización energética, necesidad de una visión holística para el cuidado. Sin embargo, este trabajo resaltó la construcción de narrativas que explicitan la pretensión de situar a esta terapia alternativa como un recurso aceptable y válido sobre la base de su inscripción en un supuesto sentido común compartido. De ahí el sentido de recorrer distintos ejes que recuperan la experiencia personal y colectiva respecto de la eficacia "objetiva" de la terapia, así como los intentos de legitimar estas experiencias a través de criterios de inteligibilidad más cercanos a los discursos "oficiales".

Retomando la discusión teórica que sustenta este trabajo, esto forma parte del proceso de "fusión de lo extraño y lo familiar para producir un híbrido local aceptable" (FADLON, 2005, p. 18, traducción propia). Nuestro aporte consiste en señalar - sin negar la incorporación de nuevos elementos que aportan medicinas y terapias de raigambre oriental - que la aceptación de ciertas alternativas supone su interpretación en términos de cercanía sociocultural. $\mathrm{Y}$ que esta familiaridad acarrea posicionamientos y actitudes de defensa. No necesariamente por la existencia de un compromiso o lazo terapéutico fuerte sino en virtud del estatuto de verdad que se le atribuye a la experiencia - propia y ajena -, central para esgrimir definiciones de sí y de sus otros significativos en términos de competencia. Consideramos que este constituye un importante aspecto en los procesos de asimilación de terapias foráneas. 


\section{Referencias}

ADAMS, G. Shiatsu in Britain and Japan: Personhood, holism and embodied aesthetics. Anthropology \& Medicine, v. 9, n. 3, p. 245-266, 2002.

BAARTS, Ch.; PEDERSEN, I. Derivative benefits: exploring the body through complementary and alternative medicine. Sociology of Health and Illness, v. 31, n. 5, p. 719733, 2009.

BARRY, C. The role of evidence in alternative medicine: Contrasting biomedical and anthropological approaches. Social Science and Medicine, n. 62, p. 2646-2657, 2006.

BORDES, M. Construcciones y transacciones de sentido en torno al cuidado de la salud en el contexto de una terapia no-convencional: la reflexología. Un estudio sobre las nuevas culturas terapéuticas en la región metropolitana de Buenos Aires. Tesis de doctorado: Facultad de Ciencias Sociales, Universidad de Buenos Aires, 2012.

. Reflexiones sobre los límites de la kinesiología desde la perspectiva de usuarios de terapias alternativas. Scripta Ethnologica, v. 29, p. 91-108, 2007.

CANT, S.; SHARMA, U. (ed.) A new medical pluralism? Alternative medicine, doctors, patients end the state. Londres: Routledge, 1999.

CAROZZI, M.J. Nueva Era y Terapias Alternativas. Buenos Aires: Universidad Católica, 2000.

CRIVOS, M. El estudio de la narrativa de casos: una propuesta para el abordaje etnográfico de las alternativas médicas. In: IDOYAGA MOLINA, A. (Comp.) Los caminos terapéuticos y los rostros de la diversidad". Buenos Aires: Espacio, 2007.

FADLON, J. Negotiating the holistic turn: the domestication of alternative medicine. Albany, NY: State University of New York, 2005.

FREIDIN, B.; TIMMERMANS, S. Complementary and alternative medicine for children's asthma: satisfaction, care provider responsiveness and networks of care. Qualitative Health Research, v. 18, p. 43-55, 2008.

GALE, N. From body-talk to body-stories: body work in complementary and alternative medicine. Sociology of Health and Illness, v. 33, n. 2, p. 237-251, 2011.

GIDDENS, A. Las nuevas reglas del método sociológico. Crítica positiva de las sociologías comprensivas. Buenos Aires: Amorrortu, 2007.

IDOYAGA MOLINA, A. Culturas, enfermedades y medicinas: reflexiones sobre la atención de la salud en contextos interculturales de Argentina. Buenos Aires: IUNA, 2002.

JOHANNESSEN, H. Individualised knowledge: reflexologists, biopaths and kinesiologists in Denmark. In: CANT, S.; SHARMA, U. (eds.) Complementary and alternative medicines. Knowledge in practice. London: Free Association, 1996. 
LAPLANTINE, F. Antropología de la enfermedad. Buenos Aires: Ediciones del Sol, 1999.

LEISER, D. Support for non-conventional medicine in Israel: cognitive and sociological coherence, Sociology of Health and Illness, v. 25, n. 5, p. 457-480, 2003.

LUZ, M. Cultura contemporânea e medicinas alternativas: novos paradigmas em saúde no fim do século XX, Physis. Rev. Saúde Coletiva. Rio de Janeiro, v. 7, n. 1, p. 13-43, 1997.

MacGREGOR, K.; PEAY, E. The choice of alternative therapy for health care. Social Science and Medicine, v. 43, n. 9, p. 1317-1327.

MAGNANI, J. G. O circuito neo-esotérico na cidade de Sao Paulo. In: CAROZZI, M. (Org.). A Nova Era no Mercosul. Petrópolis: Vozes, 1999.

MALUF, S. Mitos coletivos, narrativas pessoais: cura ritual, trabalho terapêutico e emergência do sujeito nas culturas 'Nova Era'. Mana, v. 11, n. 2, p. 499-525.

McGUIRE, M. Ritual Healing in Suburban America. New Brunswick: Rutgers University Press, 1988.

MINAYO, M. C. S. de. La artesanía de la investigación cualitativa. Buenos Aires: Lugar, 2009.

ORGANZACIÓN MUDIAL DE LA SALUD. Estrategia de la OMS sobre medicina tradicional (2002-2005). Disponible en: http://apps.who.int/medicinedocs/es/d/Js2299s/, 2002. Acceso en: 15 jun. 2008.

OTTO, R. Lo santo. Madrid: Alianza Editorial, 2005.

SAIZAR, M. De Krishna a Chopra. Filosofías y prácticas del Yoga en Buenos Aires. Buenos Aires: Antropofagia, 2009.

SCHUTZ, A. Estudios sobre teoría social II. Buenos Aires: Amorrortu, 2003.

SHARMA, U. Complementary medicine today. Practitioners and patients. London: Routledge, 1992.

SOINTU, E. Recognition and the Creation of wellbeing. Sociology, v. 40, p.493-510, 2006.

STAKE, R. Case Studies. In: DENZIN, N; LINCOLN, Y. (eds.) Handbook of Qualitative Research. California: Sage, 1994.

STEPHENSON, N.; DALTON, J. A. Using reflexology for pain management. Journal of Holistic Nursing, v.21, p.179-191, 2003.

VISACOVSKY, S. La constitución de un sentido práctico del malestar cotidiano y el lugar del psicoanálisis en la Argentina. Cuicuilco, v. 45, p. 17-47, 2009.

\section{Notas}

${ }^{1}$ Siguiendo a Idoyaga Molina (2002), denominamos "medicinas alternativas" a aquellas, principalmente de raigambre oriental, que si bien son tradicionales en otras latitudes, son de reciente incorporación en nuestro país, como el yoga, reflexología, reiki, acupuntura, ayurveda, tai chi chuan. La 
autora utiliza este concepto para distinguirlas de otras medicinas, como la medicina científica (biomedicina), las medicinas tradicionales (shamanismos indígenas y curanderismo); y religiosas (terapia católica, pentecostal, afro-umbanda, etc.).

${ }^{2}$ Esta terapia supone la estimulación manual de pies y tobillos, bajo el supuesto de que los mismos se corresponden anatómicamente con el cuerpo humano en tanto totalidad. Elegimos esta terapia por tratarse de una de las alternativas más utilizadas en nuestra área de estudio (IDOYAGA MOLINA, 2002) y en virtud de la heterogeneidad y eclecticismo que revisten las medicinas alternativas (MALUF, 2005), lo que nos condujo a seleccionar una sola en pos de reconstruir las redes de relaciones establecidas en este espacio terapéutico. Los criterios de inclusión de las unidades de análisis -usuarios y especialistas- tuvieron como objetivo captar la variabilidad, de acuerdo con diferentes niveles socioeconómicos, estilos culturales y circuitos de oferta-demanda. Como ya ha sido señalado desde la bibliografía especializada, aún no se cuenta en el área de estudio en cuestión el abordaje cuantitativo que nos permitiría dar cuenta de datos de consumo alternativo en términos poblacionales.

${ }^{3}$ De acuerdo al autor, los motivos porque hacen referencia al pasado y pueden ser denominados como razón o causa de la acción. El tipo de complejo de sentido supuesto en esta denominación difiere, así, del complejo de sentido de los motivos para - referido al futuro, idéntico al objeto o propósito para cuya realización la acción misma es un medio (SCHUTZ, 2003, p. 24).

${ }^{4} \mathrm{La}$ autora se refiere a la medicina basada en la evidencia (evidence-based medicine).

${ }^{5}$ Como señala Fadlon (2004) esto se comprende a la luz de la negociación cultural entre biomedicina y medicinas no-convencionales, en el marco de un proceso de profesionalización y búsqueda de reconocimiento oficial por parte de los practicantes de estas últimas. La autora remarca el trabajo de creación de híbridos - por ejemplo, la combinación de una técnica milenaria a través de alta tecnología como el láser; y el trabajo de traducción entre términos de distintas tradiciones culturales, como el modo en que el término "energía" pasa al campo de la psico-neuro-inmunología (FADLON, 2004, p. 82).

${ }^{6}$ Aquí, retomamos a Idoyaga Molina (2002), quien define como medicina tradicional al curanderismo y los shamanismos indígenas. 


\section{Abstract}

"This is not 'alternative' at all". The

construction of the rationality of the use of nonconventional medicine from the perspective of its users in Buenos Aires, Argentina

The study explores one aspect of non-conventional medical field (Buenos Aires): the social construction of narratives justifying the choice and use of a particular alternative therapy (reflexology) in terms of a reasonable and valid action, beyond alternative notions. The study is based on interpretive and qualitative approach, through in-depth interviews with users of this therapy. The results focus on the centrality of three themes which this narrative of reasonableness arises: (a) the experience of the "significant others", under the rhetoric of recommendation and proof; (b) specific definitions of "medical reality" from which therapy is seen as effective; and (c) one that validates therapy distinguished from other options considered radical otherness. It is proposed that this type of narrative is one of the possible expressions of acculturation and assimilation processes that cross these therapies in Western contexts, enabling acceptance from many sectors of society beyond adherence to alternative belief system.

> Key words: non-conventional medicine; reflexology; assimilation processes. 
"Isso não tem nada de 'alternativo"': a construção da racionalidade do uso de medicina não convencional na perspectiva de seus usuários em Buenos Aires, Argentina $\mathrm{O}$ artigo explora um aspecto do campo da medicina não convencional na cidade de Buenos Aires: a construção coletiva de narrativas que justificam a escolha e o uso de uma terapia particular (reflexologia) em termos de uma ação razoável e válida, baseada em sua inserção em um suposto senso comum, além da adesão a uma ideologia alternativa. $\mathrm{O}$ artigo se baseia em pesquisa de tese de doutorado, com abordagem interpretativa e qualitativa por meio de entrevistas em profundidade com usuários desta terapia. Os resultados enfocam a centralidade de três áreas temáticas em que esta narrativa da racionalidade emerge: a que se baseia na experiência dos "outros significativos", sob a retórica da recomendação e a prova; a que se relaciona com as definiçōes específicas de "real", a partir da qual a terapia é considerada eficaz; e uma que valida a terapia, distinguindo-a de outras opçôes consideradas alteridades radicais. Conclui-se que este tipo de narrativa é uma das possíveis manifestaçōes de processos de aculturação e assimilação que atravessam essas terapias em contextos ocidentais, permitindo sua aceitação por amplos setores da sociedade para além da adesão ao sistema de crenças alternativo.

> Palavras-chave: medicina não convencional; reflexologia; processos de assimilação. 